\title{
Quantitative Analysis of the Sympathetic Efferent Activity to Characterize the Integrative Function of the Sympathetic Ganglion
}

\author{
Tibor Tóth, ${ }^{*}$ Hiroshi NaKahama, Keisetsu Shima, \\ Mitsuaki Yamamoto, and Kojiro AyA \\ Division of Neurophysiology, Institute of Brain Diseases, \\ Tohoku University School of Medicine, \\ Miyagi, 980 Japan
}

\begin{abstract}
Using the concept of the Markov dependency, we analyzed the spontaneous activity in the pre- and postganglionic nerve trunks of the feline superior cervical ganglion. The time structure (order and value of time dependency) of the sequence of compound action potentials was determined. The analysis revealed that the time dependency of the postganglionic activity is of higher order and is generally more consistent than that of the preganglionic one. The difference in orders and values between the pre- and postganglionic activities was found to be a measure of the integrative function of the ganglion. A transformation by the ganglion of some additional information, inaccessible in the time structure of the preganglionic activity, seems to underlie this difference.
\end{abstract}

Key Words: sympathetic ganglion, Markov dependency, integrative function.

Since ADRIAN's (1932) description of the spontaneous electrical activity in the lapin cervical sympathetic nerve trunk, a large number of papers have been devoted to study the sympathetic discharge patterns. Periodicities linked with the cardiac and the respiratory cycles have been recognized and later verified by several analyzing techniques (see HILlaRP, 1960). The intensity and consistency of these regularities depend, however, on the actual site of recording within the sympathetic system and are subject to changes in time. It is well known, for example, that patterns of the spontaneous activity change partially or totally during somato- or viscero-sympathetic reflexes (FeDINA et al., 1966). Hence, it is obviously difficult to compare the patterns of the spontaneous and evoked activity on a common basis.

Received for publication February 23, 1982

* On leave from the Department of Experimental Physiology, National Institute of Occupational Health, Budapest, Hungary

中浜 博, 嶋 啓節, 山本光璋, 綾 皓二郎 
As for the discharge patterns of various sympathetic nerves usually the similarities are emphasized. In particular, this is valid for the activity in pre- and postganglionic sympathetic nerves of the same ganglion (SKOK, 1973; PoLOSA et al., 1979). In this case the similarities are self-evident: the postganglionic activity is generated exclusively by the preganglionic one (SKOK, 1973). In the present paper, we are going to put more emphasis on the differences between them. These differences are obviously due to the ganglionic transmission process. The latter can be approached only by studying the global properties of the whole neuron pool which are reflected in the mass discharge activity.

At present, there is hardly any doubt that sympathetic ganglia act, in some sense, as "little brains" (Winslow, cited by Brooks et al., 1979), i.e. that they operate as small integrative centers. Their integrating functions have been ascribed mainly to the convergence-divergence relations of the preganglionic fibers on the ganglion cells (Hillarp, 1960; SKoK, 1973; Polosa et al., 1979). Nevertheless, there seems to be in use, so far, no satisfactory approach that would suit the task of characterizing quantitatively the nature and the actual state of the ganglionic integratory process. Treating sympathetic activity as a Markov process offers such a possibility.

For the analysis of the sympathetic activity, correlation techniques which reveal periodic components in stochastic or quasi-stochastic processes, and various integrating and averaging techniques which yield quantitative parameters have widely been used. The latter, however, have no bearing on the analysis of the time structure. We have aimed, therefore, at description of the sympathetic activity in terms of a Markov process in order to obtain appropriate information on the intensity and consistency of time dependent patterns by determining the order and value of the Markov dependency (NAKAHAMA and Nishioka, 1966; NAKAHAMA et al., 1972, 1974, 1975, 1977, 1979). Comparing the results derived from the preand postganglionic activities we have made an attempt to assess integrative function of the superior cervical ganglion.

\section{METHODS}

Experimental technique. We performed experiments on 12 cats. The animals were anaesthetized with chloralose-urethane $(50 \mathrm{mg} / \mathrm{kg}$ and $250-300 \mathrm{mg} / \mathrm{kg}$, i. p., respectively). The pre- and postganglionic nerve trunks of the right superior cervical ganglion were prepared and the cat was fixed in a stereotaxic frame. For recording, lateral branches, e. $g$. leading to the external carotid body, were preferred, as they were long and thin enough to give good signal to noise ratio in the records. One preganglionic and one to three postganglionic trunks were always used in an experiment. The activity of the pre- and postganglionic nerve trunks (Fig. 1) was picked up by bipolar Pt-electrodes (diameter, $0.4 \mathrm{~mm}$ ) and passed through AC-amplifiers (time constant: $0.03 \mathrm{sec}$ ). The amplitude of the signals 


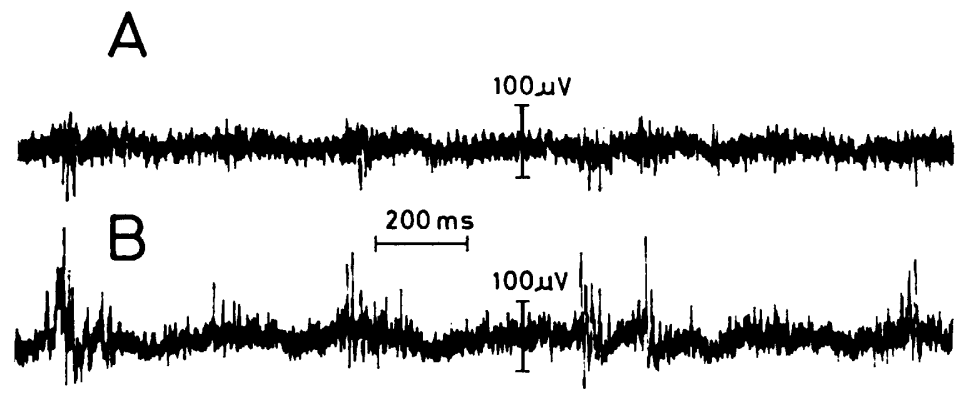

Fig. 1. Activity of the pre- and postganglionic nerve trunks recorded simultaneously. A, preganglionic; B, postganglionic activity. The inter-electrode distance was $1.5 \mathrm{~mm}$ in both cases. The total length to the segment displayed is $2,016 \mathrm{msec}$.

was between 50 and $200 \mu \mathrm{V}$. They were stored on magnetic tape in analog form using a TEAC SR-50 (FM system) recorder. For the sake of the subsequent computer-aided analysis, relatively long, continuous records of 20-60 min were made.

Data analysis. Data of three experiments showing the highest signal to noise ratios were selected for the computer-aided analysis which was carried out by means of a PDP 11/60 computer configuration. The analysis comprised two stages. First, a preprocessing was necessary in order to obtain a point process reflecting the time structure of the sympathetic activity. Then the Markov analysis of this point process was performed regarding the interpoint intervals as random variables.

Constructing such a point process is, in principle, quite simple: the time points of the peaks (regardless of the magnitude) of the compound action potentials become points of the new process. In case of bipolar recording two separate processes can be constructed. In practice, however, noise and hum are superimposed on the activity. Accordingly, discrimination of the useful signals from the noise and hum is necessary. The main problem is to find an appropriate level of discrimination at which almost all of the noise and hum are rejected without any significant loss of useful signals. If the noise and hum can be obtained in pure form, it can be effectively used to determine the optimal discrimination level. We deemed the base line to fulfill this criterion. The base line was recorded after the nerve trunk had been cut centrally. If we apply the same discrimination level to the activity and the base line, two extreme cases can occur. If this level is too low, numerous peaks of noise and hum will be recognized like useful signals even in the base line. If this level is too high, a lot of useful signals (peaks of compound action potentials) will be rejected. We applied, therefore, a series of discrimination levels both to the activity and the base line and computed the relative number of peaks over these levels. The evaluation of the computed results of all experiments showed that the relative number of peaks over the discrimination level decreased faster at low levels in the base line than in the activity. At high levels we had the 

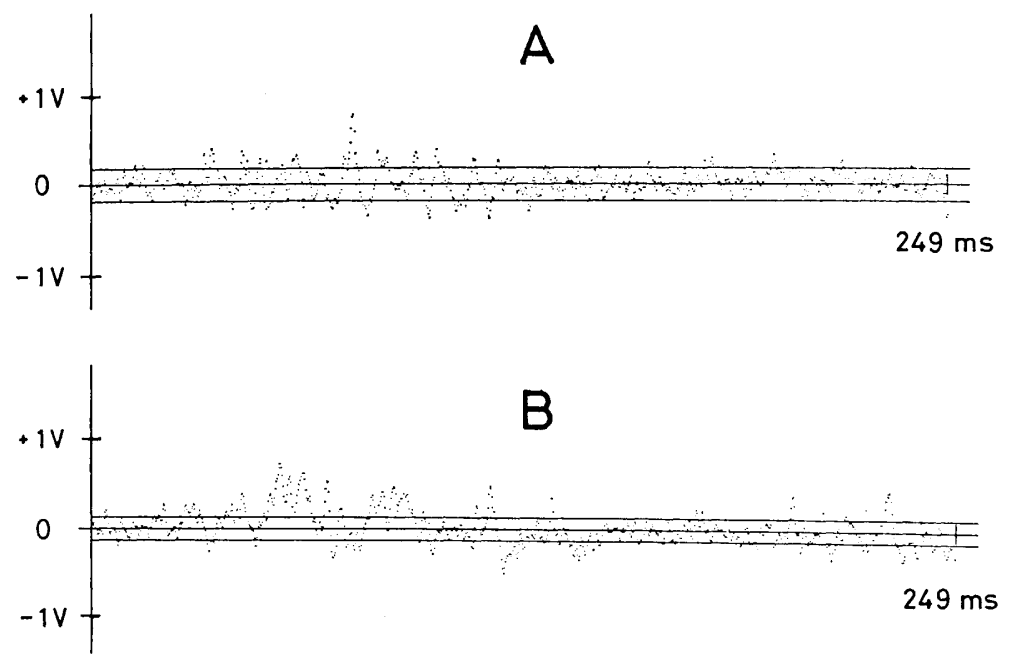

Fig. 2. Pre- and postganglionic activities displayed after digitalization and filtering for $249 \mathrm{msec}$ (900 digitalized points). A, preganglionic; B, postganglionic record. The lines parallel to the time axis are the optimal discrimination levels (see text): $2 \sigma$ with $\sigma=$ $0.091 \mathrm{~V}$ and $1.6 \sigma$ with $\sigma=0.076 \mathrm{~V}$ for $\mathrm{A}$ and $\mathrm{B}$, respectively.

opposite case. It means that at low levels the effectivity of noise rejection increases while at high levels useful signals, too, are increasingly lost. When the relative number of peaks over the discrimination level in the activity became about two times as large as that in the base line, the rates of decrease of both parameters were approximately equal. In this case, peaks in the base line were rarely greater than the discrimination level. On the other hand, this level was not yet so high that a considerable number of useful signals in the activity would have been rejected. Therefore, this level has been regarded as optimal.

The preprocessing was begun with the digitalization of the analog data by means of a 12 bit A/D converter at a sampling rate of $3.611 \mathrm{kHz}$. Pre- and postganglionic activities were handled simultaneously. All the digitalized records had been processed with a symmetrical digital filter of order 7 provided by the computer software, and then the average values and the standard deviations were computed. The magnitude of the discrimination level was measured from the average value of the actual biphasic signals (of the activity and of the base line, respectively) having the standard deviation of the base line as the unity (Fig. 2). Figure 3 shows a short segment of the digitalized signals displaying the construction principle of the point process. As mentioned above, two point processes could be constructed from the sympathetic activity in the ideal case. Because of asymmetries in the records, however, only one of them was usually of satisfactory quality after the procedure.

Having determined the optimal levels of discrimination we carried out the 


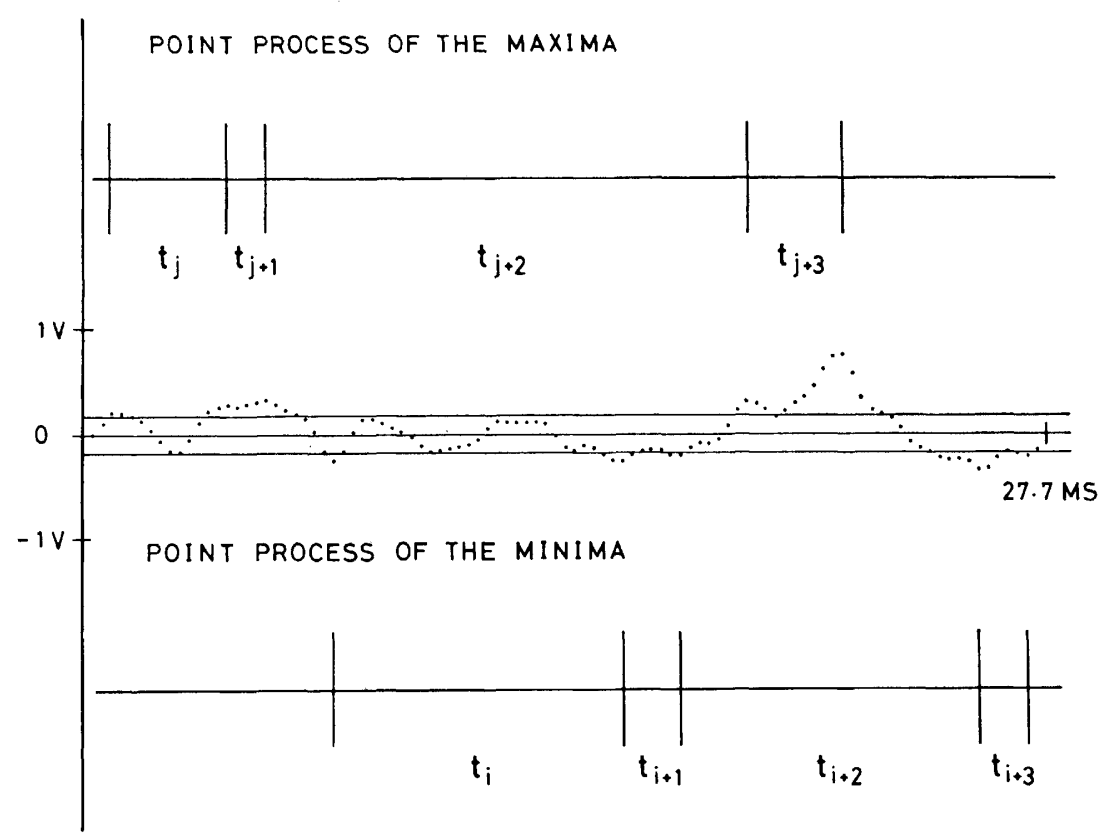

Fig. 3. The construction principle of the point process. Middle trace, a $27.7 \mathrm{msec}(100$ points) long segment of the preganglionic record from Fig. 2 (from point 301 to 400 ); top, the process of the local maxima; bottom, that of the local minima. $t_{i}$ and $t_{j}$ denote the lengths of the intervals. The discrimination level is the same as in Fig. 2.

analysis to verify the presence of Markov properties. The concept of Markov properties and the method to verify the presence of Markov properties in nerve impulse trains have been developed by NAKAHAMA and NishioKA (1966). A short theoretical outline of this method is given in APPENDIX of this paper. In our analysis we used the concept of simplified dependency.

In order to select stationary segments of records for analysis we applied the $U$-test by Cox and LewIS (1966) to the point processes obtained from the pre- and postganglionic activities.

\section{RESULTS}

In all cases analyzed, the preganglionic activity showed orders of dependency varying from 0 to 3 while those of the postganglionic one varied from 4 to 8 . The pairs of corresponding orders for the pre- and postganglionic activities recorded simultaneously were as follows: $0-4,2-5,3-5,2-6,3-6,2-7,3-7$, and 3-8. A typical case is shown in Fig. 4. Results of the analysis within a particular experiment remained rather consistent, if the time period was sufficiently long (about $50 \mathrm{sec}$ ) and the sample size of the intervals was large enough (about 4,000 for the pre- and 9,000 for the postganglionic data). In short segments of 1,000 intervals 

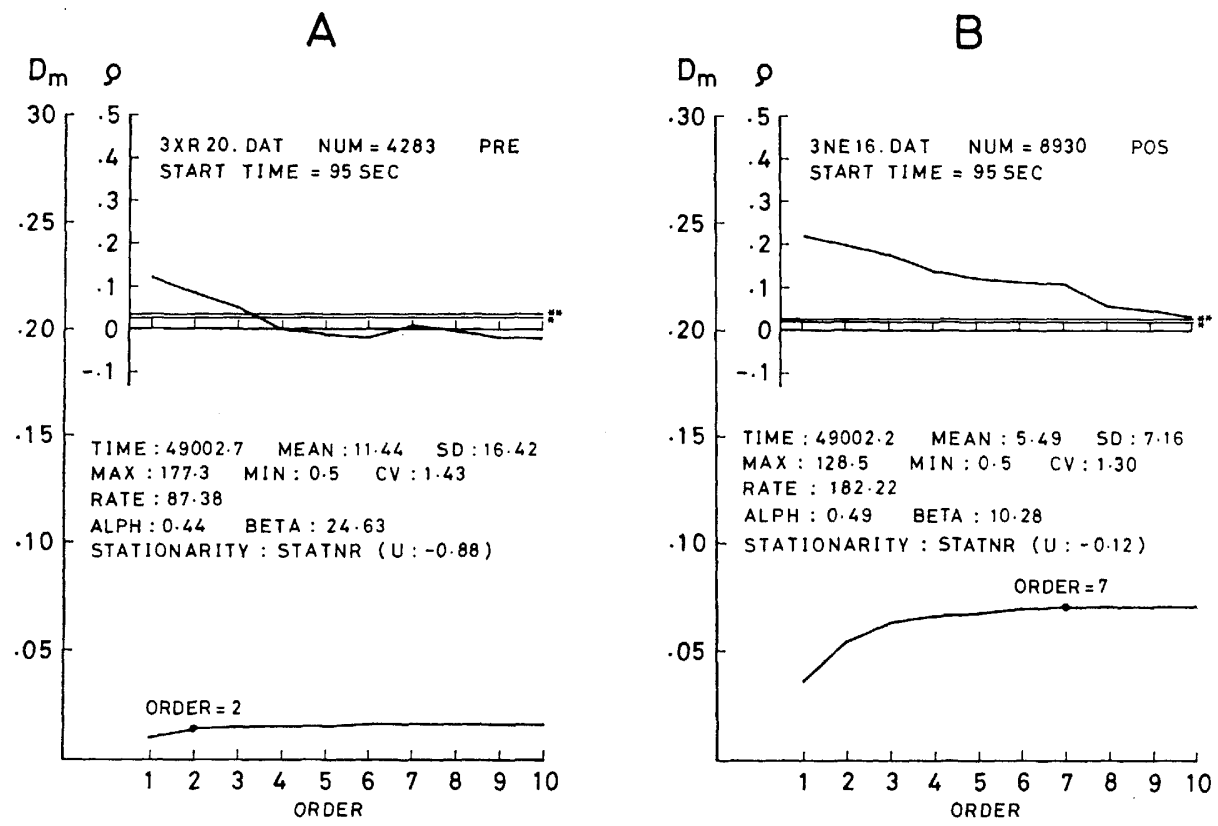

Fig. 4. Values of dependency and serial correlation coefficients. A, preganglionic activity (PRE); B, postganglionic activity (POS). 3XR20. DAT, 3NE16. DAT, names of the data files; NUM, number of analyzed intervals; START TIME, length of the time interval from the beginning of the file up to the starting point of the analysis. Large coordinate system: value of dependency $v s$. order of dependency. The order of the actual process is estimated by Eq. (17) where a significance level is 0.01 , being indicated on the dependency curve. Small coordinate system: value of the serial correlation coefficients $v s$. order. The two horizontal lines indicated with * and ** show significance levels of 0.05 and 0.01 , respectively. For negative valued coefficients negative significance levels symmetrical to the horizontal axis are valid. TIME, duration of the analysis; MEAN, mean value; SD, standard deviation; MAX and MIN, maximal and minimal value of the analyzed intervals respectively. All of these variables are given in milliseconds. $C V=S D / M E A N$; $\mathrm{ALPH}=(\mathrm{MEAN}-\mathrm{MIN})^{2} / \mathrm{SD}^{2} ; \mathrm{BETA}=\mathrm{SD}^{2} /(\mathrm{MEAN}-\mathrm{MIN})$. RATE is the average number of discharges per second (RATE $=1000 / \mathrm{MEAN}$ ). The value $U$ shows whether the process is stationary. If $U \leqq 1.96$ it is considered to be stationary.

we found that the postganglionic signals had a higher order and a larger value of dependency (Fig. 5). The dependency order of short segments, however, was always lower than that of the longer ones. Segments of 45-50 sec in length sufficed to yield reliable dependency orders.

The optimal discrimination levels were usually about 2.0 and 1.6 times of the standard deviation of the corresponding base lines for the pre- and postganglionic activities, respectively. In a single experiment these levels amounted to 2.6 and 1.8 indicating a worse signal to noise ratio in the records. Accordingly, the order (and the value) of the Markov dependency proved to be less ( 0 and 4 for the pre- 
A

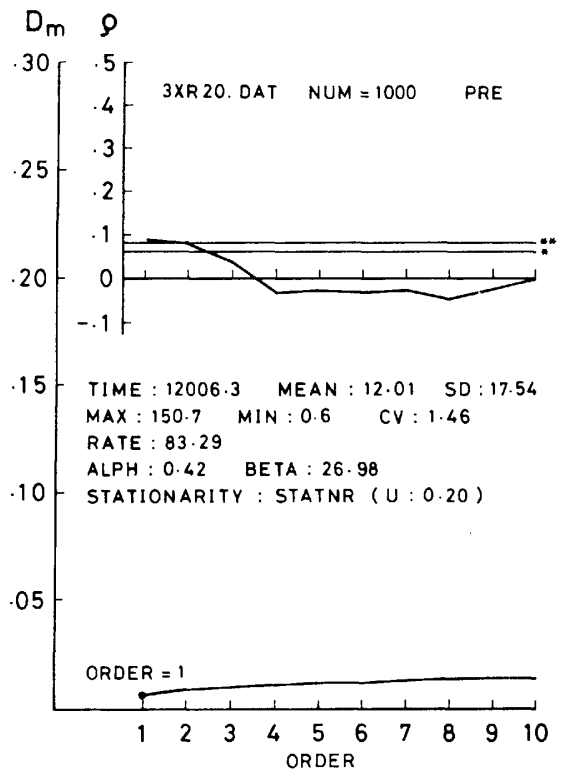

$\mathrm{B}$

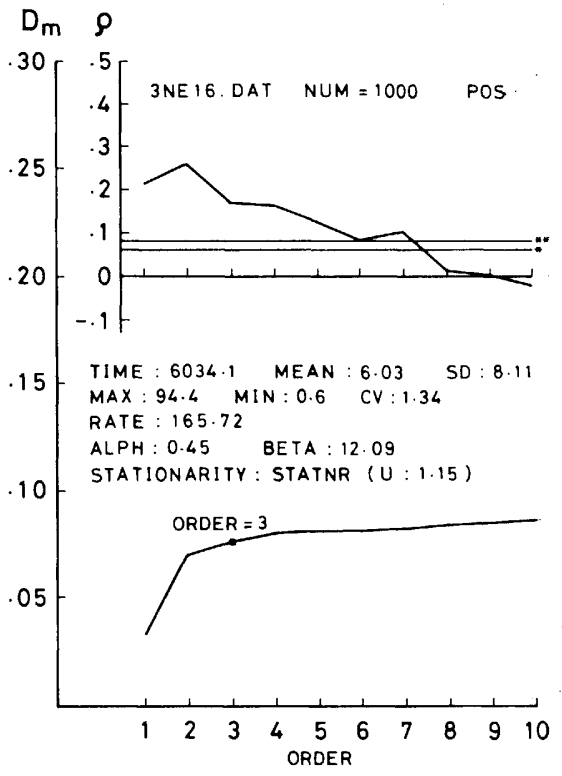

Fig. 5. Values of dependency and serial correlation coefficients of a segment of 1,000 intervals. All notations are the same as in Fig. 4. Note that the segments analyzed are not equal, the preganglionic being about twice the length of the postganglionic one.

and postganglionic activity, respectively) than in other experiments with lower optimal discrimination levels. Nevertheless, the relation between the dependency orders of the pre- and postganglionic activities remained essentially the same. Generally, the order and the value of the Markov dependency decrease if the discrimination level increases.

The dependency value of the preganglionic activity remained below 0.05 in any case while that of the postganglionic one increased above this value (Fig. 4); it reached sometimes even 0.1 . In both cases the slope of the dependency curve decreased monotonically. This points out that the influence of the preceding intervals gradually diminished with their order of precedency.

The serial correlation coefficients being in close relation to the dependency order and value are shown in Fig. 4. Neither of the pre- and postganglionic activities possessed significant negative correlation (Figs. 4 and 5). This is generally true for all the data analyzed. In accord with the dependency parameters the values of the serial correlation coefficients computed from the postganglionic activity exceeded always those of the preganglionic ones.

In order to provide further information of the pre- and postganglionic activities the corresponding interval diagrams and histograms are shown in Fig. 6. The magnitudes in the interval diagram display the lengths of time intervals in loga- 


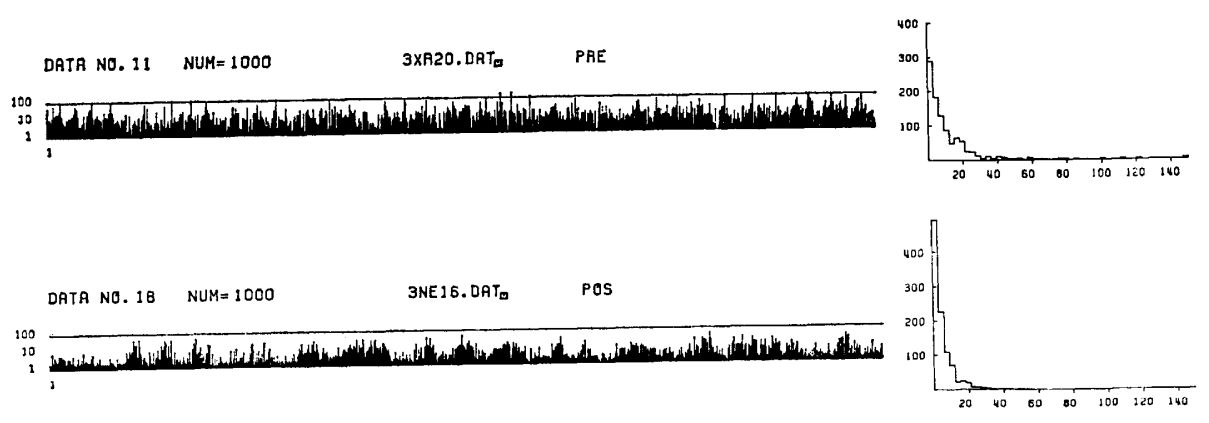

Fig. 6. The interval diagram and histogram of the pre- and postganglionic activities. Top, preganglionic; bottom, postganglionic data. In the interval diagram on the left side each vertical line means the length of an interval in logarithmic scale in milliseconds, the $100 \mathrm{msec}$ level is indicated by a horizontal line. Horizontal axis: ordinal numbers of the intervals from 1 to 1,000 . The histograms of the same 1,000 intervals have a bin width of $3 \mathrm{msec}$ and a range up to $150 \mathrm{msec}$.

rithmic scale. Figure 6 provides visual information of the regularities in the activities. The histogram is to be considered as an approximation of the probability density function of the distribution of the time intervals. The knowledge of the density function is important, because there are some special distributions to which the method of the simplified dependency cannot be applied (NAKAHAMA et al., 1977). As it can be seen from the histograms, we had exponential distributions and, therefore, no restrictions appeared in applying the simplified dependency method. Moreover, the interval diagram and histogram and the basic statistics shown in Figs. 4 and 5 establish a link between the original physiological data and the rather abstract results of the dependency analysis.

\section{DISCUSSION}

Applying the simplified dependency method to the mass discharge activity of the sympathetic efferent nerve trunks, we could obtain a classification of its patterns in terms of a Markov process. The parameters are the order $m$ and the value $D_{k}(k=1, \cdots, m)$ of the time dependency of the process. In other words the system producing the process $x_{t}$ has a "memory" of order $m$, since $m$ preceding intervals have an influence on an interval under consideration. The dependency value determines this influence quantitatively through the differences (NAKAHAMA et al., 1972, 1974, 1977).

$$
\Delta D_{k}=D_{k}-D_{k-1} \quad(k=1, \cdots, m),\left(D_{0}=0\right) .
$$

Furthermore, there is a correspondence between the dependency value $D_{k}$ and the conditional distributions of order $k$ (see APPENDIX). If the conditional distributions are sharp, $i$. e. for one particular $l$ the values of the probability is much greater than any other of the same combination $j_{1}, \cdots, j_{k}$, the value of $D_{k}$ will become rather 
high. In the extreme case, when $p\left(l \mid j_{1}, \cdots, j_{k}\right)=1$ is valid for some $l_{0}$ and $j_{1}, \cdots$, $j_{k}, D_{k}$ will reach the value of 1 (NaKahama $e t$ al., 1977). Hence, the greater the frequency of occurrence of some particular combinations compared with all the remaining ones, the higher is the value of dependency. We consider these combinations as the regular patterns of the process. Accordingly, we can conclude that the higher the value of dependency the higher is the consistency of these patterns. The dependency value, due to the correspondence between the point process and sympathetic activity, can be regarded as a measure for the consistency or reproducibility of the patterns present in the activity.

The dependency analysis has shown a rather weak and inconsistent regularity in the preganglionic activity, whereas a strong and consistent one in the postganglionic activity. This is in accordance with the generally accepted fact that in the latter the rhythmic fluctuations linked with cardiac and respiratory cycles are amplified and are thus better to recognize (Fig. 1) and seem to be more consistent (SKoK, 1973; PolosA et al., 1979).

Since the results of the dependency analysis of the pre- and postganglionic activities were obtained with high reliability, they allow us to draw some conclusions concerning the integrative function of the superior cervical ganglion and, on the basis of the morphological and functional analogies, of other sympathetic ganglia, too. First of all, we can characterize this function by comparing pre- and postganglionic parameters. We have seen that the postganglionic patterns have more complex structure than the preganglionic ones, as indicated by the differences of the dependency order of 2 to 5 . The postganglionic patterns are of higher consistency, too, as shown by the dependency values. Based on these findings we propose the transganglionic transformation of the dependency parameters to be considered as an integration property of the ganglion. Accordingly, the larger the difference between the orders of dependency is and the higher the (post- to preganglionic) ratio of the dependency values is, the higher is the degree of integration by the ganglion of the activity inflow.

Of course, under some conditions the postganglionic activity might have a lower order than the preganglionic one. In such cases, one could say the ganglion disintegrates the preganglionic activity. In a single experiment, the activity of one of the postganglionic nerves showed lower dependency value compared with that of the preganglionic one, although the orders were 5 and 3 , respectively. In this case, the integration process seems to have been disturbed by some intraganglionic event (e.g. changes in the level of critical depolarization or catecholamine concentration, etc.) causing irregularities in the transmission.

The question as to where the orderliness of higher degree in the postganglionic activity come from has remained still open. The increase in the order may have its origin only in some additional source other than the time structure of the preganglionic activity. This additional type of information may be carried by the spatial configuration of the activities in individual fibers at the input of the ganglion. 
If so, the ganglion transforms spatially coded information into time structured one.

SkoK (1973) pointed out three main physiological functions of the autonomic sympathetic ganglia: multiplication of centrifugal impulses, integration of the impulse inflow and controlling peripheral reflex activity. In the opinion of PoLOSA et al. (1979), ganglionic function implies "amplification of tonic activity." The control of the peripheral reflex activity is not concerned by our results. As for the multiplication or amplification, our findings show that, beyond an increased amount frequency of the postganglionic compound action potentials, also the time dependency of the activity and the consistency of its regular patterns are enlarged by the ganglion. Thus, multiplication and amplification should cover these events. The multiplication or amplification, in this wider sense, appears as the realization of the ganglionic integratory process.

\section{APPENDIX}

One of the main objectives of dependency analysis is to infer the order and value of Markov process from the observed series of events. For this purpose, we have proposed dependency as one of statistical measures representing Markovian characteristics (NAKAHAMA and NishIOKA, 1966; NAKAHAMA et al., 1972, 1974, 1975, 1977, 1979).

The $k$-th order dependency $D_{k}$ for discrete variables in the population is defined by

$$
D_{k}=\frac{H_{0}-H_{k}}{H_{0}},
$$

where $H_{0}$ and $H_{k}$ are Shannon's entropy and the $k$-th order conditional entropy, respectively (SHANNON and WEAVER, 1949). That is,

and

$$
H_{0}=-\sum_{l=1}^{n_{s}} p(l) \log _{2} p(l)
$$

$$
H_{k}=-\sum_{j_{1}=1}^{n_{s}} \cdots \sum_{j_{k}=1}^{n_{s}} \sum_{l=1}^{n_{s}} p\left(j_{1}, \cdots, j_{k}, l\right) \log _{2} p\left(l \mid j_{1}, \cdots, j_{k}\right),
$$

where $p(l), p\left(j_{1}, \cdots, j_{k}, l\right)$ and $p\left(l \mid j_{1}, \cdots, j_{k}\right)$ are a probability of state $l$, a joint probability of state $\left(j_{1}, \cdots, j_{k}, l\right)$ and a conditional probability of state $l$ when state $\left(j_{1}, \cdots, j_{k}\right)$ is given, respectively. Here we consider that the state space $S$ is finite and consists of the first $n_{s}$ positive integers, $S=\left\{1,2, \cdots, n_{s}\right\}$, where $n_{s}$ is a total number of states in the system.

When we describe the necessary and sufficient condition of the $k$-th order Markov process by dependency, we obtain the following expression (NAKAHAMA et al., 1972):

$$
0 \leq D_{1} \leq D_{2} \cdots \leq D_{k-1}<D_{k}=D_{k+1}=D_{k+2}=\cdots \leq 1 .
$$

The order of Markov process is determined by using Eq. (4). $D_{k}$ is a monotonic 
increasing function of $k$. Let $\Delta D_{k}$ denote the increment of $D_{k}$ concerning $k$ :

$$
\Delta D_{k}=D_{k}-D_{k-1},
$$

where $D_{0}=0$. When $\Delta D_{k}=0$ for all $k \geqq k_{0}+1$ the least value of $k_{0}$ gives the order of Markov process. Here we designate the value $\Delta D_{k}$ as Markov value of order $k$. The range of $D_{k}$ covers the values between 0 and 1 for all $k$ :

$$
0 \leq D_{k} \leq 1 .
$$

In the case of independent process,

$$
D_{k}=0 \quad(k=1,2, \cdots) .
$$

In the case of completely dependent process,

$$
D_{k}=1 \quad(k=1,2, \cdots) .
$$

$D_{k}=0$ is valid if and only if $H_{0}=H_{k}$ (NAKahama et al., 1972). Then

$$
p\left(l \mid j_{1}, \cdots, j_{k}\right)=p(l) \text { for all } j_{1}, \cdots, j_{k}, l .
$$

And $D_{k}=1$ if and only if $H_{k}=0$. Then we have for some $l_{0}$ and each combination $j_{1}, \cdots, i_{k}$

$$
p\left(l_{0} \mid j_{1}, \cdots, j_{k}\right)=1 \quad \text { and } \quad p\left(l \mid j_{1}, \cdots, j_{k}\right)=0 \quad \text { for all other } l .
$$

If Eq. (9) is satisfied, the state $l$ of the process do not depend on the preceding $k$ states. If Eq. (10) is satisfied, it means that $l_{0}$ is completely determined by the preceding states and therefore totally dependent on them. Accordingly, it makes sense to call $D_{k}$ as dependency value. Thus $D_{k_{0}}$ is a statistical measure which represents degree of dependency of Markov process as a whole.

In the following we introduce dependency for continuous variables. The numerator of $D_{k}$ given by Eq. (1) represents mutual information, which increases monotonically and converges to mutual information in continuous space when $n_{\mathrm{s}} \rightarrow \infty$. On the other hand the denominator of Eq. (1) $H_{0}$ diverges in value as $n_{\mathrm{s}} \rightarrow \infty$. Accordingly we define mutual information as dependency for continuous variables (NAKAHAMA et al., 1977):

$$
\begin{aligned}
D_{k}{ }^{\prime} & =H_{0}-H_{k} \quad\left(n_{s} \rightarrow \infty\right) \\
& =Z_{1}+Z_{k}-Z_{k+1},
\end{aligned}
$$

where $Z_{k}$ is joint entropy. In the case of the $k$-dimensional Gaussian distribution, joint entropy is given by

$$
Z_{k}=\log _{2}(2 \pi \mathrm{e})^{k / 2}\left|\sigma_{i j}^{k}\right|^{1 / 2},
$$

where $\left|\sigma_{i j}^{k}\right|$ is the determinant of the covariance matrix $\left(\sigma_{i j}^{k}\right)$ (SHANnon and WEAVER, 1949). That is, $\left|\sigma_{i j}^{k}\right|=\sigma^{2 k}\left|S_{k}\right|$ for stationary process, where $\left|S_{k}\right|$ is the determinant of matrix $S_{k}$ : 


$$
S_{k}=\left[\begin{array}{lllll}
1 & \rho_{1} & \cdots & \rho_{k-2} & \rho_{k-1} \\
\rho_{1} & 1 & \cdots & \rho_{k-3} & \rho_{k-2} \\
\vdots & \vdots & & \vdots & \vdots \\
\rho_{k-1} & \rho_{k-2} & \cdots & \rho_{1} & 1
\end{array}\right],
$$

where $\sigma$ and $\rho_{i}$ are standard deviation and the $i$-th order serial correlation coefficient, respectively. Then Eq. (11) reduces to the following:

$$
\mathscr{D}_{k}=\frac{1}{2}\left\{\log _{2}\left|S_{k}\right|-\log _{2}\left|S_{k+1}\right|\right\},
$$

where we replace $D_{k}{ }^{\prime}$ by $\mathscr{D}_{k}$ for the Gaussian distribution, and designate $\mathscr{D}_{k}$ as simplified dependency. Therefore an estimate of simplified dependency, $\hat{\mathscr{D}}_{k}$, is given by

$$
\hat{\mathscr{D}}_{k}=\frac{1}{2}\left\{\log _{2}\left|\hat{S}_{k}\right|-\log _{2}\left|\hat{S}_{k+1}\right|\right\} .
$$

Markov values of order $k$ is estimated by

$$
\Delta \hat{\mathscr{D}}_{k}=\hat{\mathscr{D}}_{k}-\hat{\mathscr{D}}_{k-1} \text {. }
$$

The null hypothesis for the test of the order of Markov process is given by $\Delta \mathscr{D}_{k}=\mathscr{D}_{k}-\mathscr{D}_{k-1}=0$. The critical value $\Delta \mathscr{D}_{k}{ }^{\alpha}$ for a significance level $\alpha$ is given by the use of $\chi_{1}^{2}(\alpha)$ :

$$
\begin{aligned}
\Delta \mathscr{D}_{k}{ }^{\alpha} & =-\frac{1}{2} \log _{2}\left(1-\frac{\chi_{1}{ }^{2}(\alpha)}{N}\right) \\
& =K \chi_{1}^{2}(\alpha) / N,
\end{aligned}
$$

where $K=0.5 \log _{2} \mathrm{e}=0.72135, N$ is sample size, and $\chi_{1}^{2}$ is a random variable which has the chi-square distribution with one degree of freedom (NAKAHAMA et al., 1977).

The monotonic increasing relation of $\mathscr{D}_{k}$ holds also for the $k$-th order Markov process:

$$
0 \leq \mathscr{D}_{1} \leq \mathscr{D}_{2} \leq \cdots \leq \mathscr{D}_{k-1}<\mathscr{D}_{k}=\mathscr{D}_{k+1}=\mathscr{D}_{k+2}=\cdots
$$

However $\mathscr{D}_{k}$ could have a larger value than 1.0 , because the value of $\mathscr{D}_{k}$ is not standardized to unity.

This paper was prepared while Mr. Tóth stayed here from Hungary on a Japanese Government (Monbusho) scholarship. We would like to express our thanks to the authorities of both countries making possible our cooperation.

Furthermore, we are indebted to Dr. Akio Sato, Tokyo, Dr. Attila Mitsányi and Dr. Andor Erdélyi, Budapest, for their kind and valuable advice during the preparation of the manuscript.

\section{REFERENCES}

Adrian, E. D. (1932) The Mechanisms of Nervous Action, Oxford University Press, London, $103 \mathrm{pp}$. 
Brooks, C. McC., Uchizono, K., and Uono, M. (1979) The development of our knowledge of the autonomic nervous system. In: Integrative Functions of the Autonomic Nervous System, ed. by Brooks, C. McC., Koizumi, K., and Sato, A. Tokyo Univ. Press-Elsevier/ North Holland, Tokyo, pp. 473-496.

Cox, D. R. and Lewis, P. A. W. (1966) The Statistical Analysis of Series of Events, John Wiley \& Sons, New York, p. 285.

Fedina, L., Katunskit, A. Ya., Khayutin, V. M., and Mitsányi, A. (1966) Response of renal sympathetic nerves to stimulation of afferent $\mathrm{A}$ and $\mathrm{C}$ fibres of tibial and mesenterial nerves. Acta Physiol. Acad. Sci. Hung., 29: 157-175.

Hillarp, N.-A. (1960) Peripheral autonomic mechanisms. In: Handbook of Physiology, Section I, Neurophysiology, Vol. 2, ed. by Field, J., Magoun, H. W., and Hall, V. E. American Physiological Society, Washington, D. C., pp. 979-1006.

Nakahama, H., Aya, K., Yamamoto, M., FujII, H., and Shima, K. (1979) Dependency representing Markov properties of nonstationary spike trains recorded from the cat's optic tract fibers. Biol. Cybern., 35: 43-54.

Nakahama, H., IshiI, N., and Yамамото, M. (1972) Markov process of maintained impulse activity in central single neurons. Kybernetik, 11: 61-72.

Nakahama, H., IshiI, N., Yamamoto, M., and FujII, H. (1974) Statistical inference on Markov process of neuronal impulse sequences. Kybernetik, 15: 47-64.

NaKahama, H., Ishit, N., Yamamoto, M., Fuji, H., and Obata, T. (1975) Statistical dependency as a measure to evaluate Markov properties of stochastic point processes. Biol. Cybern., 18: 191-208.

NAKahama, H. and Nishioka, S. (1966) Statistical dependency between intervals in neuronal impulse sequences. J. Theor. Biol., 12: 140-146.

Nakahama, H., Yамамoto, M., IshiI, N., FuJII, H., and Aya, K. (1977) Dependency as a measure to estimate the order and the value of Markov processes. Biol. Cybern., 25: 209_ 226.

Polosa, C., Mannard, A., and Laskey, W. (1979) Tonic activity of the autonomic nervous system: Functions, properties, origins. In: Integrative Functions of the Autonomic Nervous System, ed. by Brooks, C. McC., Koizumi, K., and Sato, A. Tokyo Univ. PressElsevier/North Holland, Tokyo, pp. 342-354.

Shannon, C. and WeAver, W. (1949) The Mathematical Theory of Communication, Univ. of Illinois Press, Urbana, p. 117.

Sкок, V. I. (1973) Physiology of Autonomic Ganglia, Igaku Shoin, Tokyo, p. 197.

Vol. 33, No. 1, 1983 